\title{
Birds invest wingbeats to keep a steady head and reap the
}

\section{ultimate benefits of flocking}

3

4 Lucy A. Taylor ${ }^{1 *}$, Dora Biro ${ }^{1}$, Ben Lambert ${ }^{1,2}$, James A. Walker ${ }^{1}$, Graham K. Taylor ${ }^{1}$ and

Steven J. Portugal ${ }^{3}$

6

7 Affiliations:

$8{ }^{1}$ Department of Zoology, University of Oxford, South Parks Road, Oxford, OX1 3PS, UK.

$9 \quad 2$ Department of Infectious Disease Epidemiology, Imperial College London, St Mary’s

10 Campus, Norfolk Place, London W2 1PG

$113^{3}$ School of Biological Sciences, Royal Holloway, University of London, Egham, Surrey,

12 TW20 0EX, UK.

13

14 "Correspondence to: lucy.taylor@zoo.ox.ac.uk 


\section{Abstract}

18 Flapping flight is the most energetically demanding form of sustained forwards locomotion

19 that vertebrates perform. Flock dynamics therefore have significant implications for energy

20 expenditure. Despite this, no studies have quantified the biomechanical consequences of flying

21 in a cluster flock relative to flying solo. Here, we compared the flight characteristics of homing

22 pigeons (Columba livia) flying solo and in pairs, using high-precision $5 \mathrm{~Hz}$ GPS and $200 \mathrm{~Hz}$

23 tri-axial accelerometer biologgers. Paired flight increased route accuracy by $\sim 7 \%$, but, was

24 accompanied by an increase in wingbeat frequency of $\sim 18 \%$. As expected, paired individuals

25 benefitted from improved homing route accuracy, which reduced flight distance by $\sim 7 \%$ and time by $\sim 9 \%$. However, realising these navigational gains involved substantial changes in flight

27 kinematics and energetics. Both individuals in a pair increased their wingbeat frequency by c. $18 \%$, by decreasing the duration of their upstroke. This sharp increase in wingbeat frequency caused just a 3\% increase in airspeed, but reduced the oscillatory displacement of the body by $30 \sim 22 \%$, which we hypothesise relates to an increased requirement for visual stability and manoeuvrability when flocking. Overall, the shorter flight distances and increased wingbeat

32 frequency in a pair resulted in a net increase in the aerodynamic cost of returning home, which

33 we estimate was $\sim 14 \%$. Our results demonstrate that flocking costs have been underestimated

34 by an order of magnitude and force reinterpretation of their mechanistic origin. We show that,

35 for pigeons, two heads are better than one, but keeping a steady head necessitates energetically costly kinematics.

38 Key words: accelerometer, biologging, energy, flocking, pigeon, stabilisation, wingbeat

39 frequency 
Introduction

42 Across the animal kingdom many species travel in groups, from pairs to flocks, shoals, herds and swarms, some containing millions of individuals [1,2]. Indeed, the collective motion of animals produces some of the most spectacular displays of synchronisation and coordination in the world [3]. Commonly cited benefits of collective travel include an improved ability to detect and avoid predators $[1,4]$, enhanced orientational efficiency through the pooling of navigational knowledge [5-8], and energetic efficiencies derived from fluid dynamic interactions [9-13]. Flocking in birds, in particular, has received considerable attention due to the complex aerodynamic interactions that take place between group members [11-14].

Avian flock formations can be categorised as either line formations or cluster formations $[15,16]$. Line formations, which include the distinctive ' $\mathrm{V}$ ' of many long-distance migrants, are utilised by medium to large-sized birds, such as northern bald ibis (Geronticus eremita) and Canada geese (Branta canadensis), whereas cluster formations are typically observed in smaller birds, such as homing pigeons (Columba livia) and common starlings (Sturnus vulgaris), which fly in irregular three-dimensional flocks [11-16]. Birds flying in close cluster flocks in particular are able to move with near perfect synchrony, whilst making rapid directional changes in three dimensions. While birds travelling in V-formation can save energy by flying in aerodynamically optimal positioning within the $\mathrm{V}$ [11-13], those species flying in

60 cluster flocks have been shown to incur an additional energetic cost in denser formations [14].

61 In homing pigeons, for example, a tenfold increase in the spatial density of a flock has been

62 observed to be associated with a modest $0.1 \mathrm{~Hz}$ increase in wingbeat frequency, and was

63 presumed to be accompanied by an energetic cost over the seven flights that were observed 64 [14]. Flapping flight is the most energetically demanding form of sustained forwards 
locomotion that vertebrates perform [17,18], and flock dynamics may therefore have studies have yet compared the biomechanical consequences of flying in a pair to flying solo, so the energetic impact of this form of flocking is unknown.

To fill this fundamental gap, we recorded the body accelerations associated with every wingbeat of 20 free-flying homing pigeons flying solo and in pairs as they homed from a site $7 \mathrm{~km}$ east of their loft (Fig. 1A). The birds were equipped with $5 \mathrm{~Hz}$ GPS trackers and $200 \mathrm{~Hz}$ tri-axial accelerometer biologgers which allowed us to reconstruct their trajectories and wingbeat patterns during each homeward flight (see Methods; Fig. 1B-F) $[19,20]$. The experiment consisted of four phases. In Phase 1, each subject first completed 21 successive solo flights (Fig. 1F), the last six of which provided the solo baseline (solo 1). During these six solo flights, the median wingbeat frequency and amplitude (dorsal body displacement) for all birds were $5.48 \pm 0.19 \mathrm{~Hz}$ (grand mean of the median value of each flight \pm s.d. of the individual means) and $20.68 \pm 1.17 \mathrm{~mm}$, respectively (after accounting for the effects of airspeed, date of release and weather variables including wind support, crosswind, temperature, humidity and

81 air density; Fig. 2A). These wingbeat frequencies are consistent with those measured

82 previously for solo pigeon flights [20,21]. In Phase 2, following the solo releases, birds were 83 released six times from the same - now familiar - site, but in similar-sized pairs. Pairs were 84 assigned based on similarity in body mass and structural size as measured by tarsus length [22].

85 Body size and mass are strong predictors of preferred flight speeds in birds, both at the intraand inter-specific levels, with optimal flight speeds usually assumed to be those for which the cost of transport (i.e. energy expenditure per unit distance) is predicted to be at its minimum [18]. Therefore, we hypothesised that for birds of different sizes either one or both birds may have to adjust their wingbeat frequency and/or amplitude to stay together as a pair, which 
would represent an additional 'hidden' compromise cost of flying with another bird. In Phase

913 , immediately following completion of the six similar-sized pair releases, each bird was then

92 flown in size-mismatched pairs for a further six flights (different-sized pairs), again from the

93 same site. Finally, in Phase 4, upon completion of the six size-mismatched flights, each bird

94 flew six times solo again (Fig. 2D). We compared the wingbeat characteristics of birds flying in pairs relative to flying solo to determine if pigeons alter their wingbeat characteristics when flying in a pair.

97

\section{Results}

99

100 We analysed the data from all flights using Bayesian hierarchical models to account for

101 variation due to a set of environmental covariates, and the individual identity of the focal bird and any partner (see Methods). Our results show that when birds flew in pairs, their median wingbeat frequency increased by $1.00 \mathrm{~Hz}$ (95\% Bayesian credible interval (CrI) [0.61, 1.38]) relative to flying solo, representing an increase of $18.2 \%$ (Fig. 2B-C). This was not associated with greater variability in wingbeat frequency, the standard deviation of which remained stable between solo and paired flight ( $2 \%$ lower standard deviation for size-matched pairs). Likewise, the median peak-to-peak amplitude of dorsal body (DB) acceleration was similar in both solo and paired flights (difference of $0.16 \mathrm{~g}$, where $\mathrm{g}$ is gravitational acceleration; $95 \% \mathrm{CrI}[-0.16$, 0.47], where the fact that the credible interval crosses zero indicates that any difference is

110 statistically indistinguishable from zero). This combination of increased wingbeat frequency

111 and unchanged amplitude of DB acceleration resulted in a net $22.5 \%$ reduction in the median

112 peak-to-peak amplitude of dorsal body (DB) displacement (-4.65 mm, 95\% CrI [-6.31, -3.06])

113 through the wingbeat, as a result of the correspondingly shorter time period over which DB 
114 acceleration is integrated to produce DB displacement (see supplementary text for further

115 analysis of the oscillatory accelerations experienced by an accelerometer).

117 These statistical findings were consistent within and between pairs, irrespective of whether the

118 birds were flying in similar-sized or different-sized pairs $(0.01 \mathrm{~Hz}$ increase per $\mathrm{mm}$ difference

119 in tarsus length between the pair; 95\% CrI [-0.13, 0.16]; Fig. S1), or whether the bird was in

120 front or behind during the flight $(0.01 \mathrm{~Hz}$ increase for travelling behind, $95 \% \mathrm{CrI}[-0.10,0.12])$.

121 Moreover, the probability of whether an individual bird flew ahead in a given pair was

122 unaffected by the birds' tarsus length (0.01 per mm difference in tarsus length, $95 \% \mathrm{CrI}[-2.68$,

$1232.44])$, solo airspeed (-0.22 per $\mathrm{m} \mathrm{s}^{-1}$ difference in median solo airspeed, 95\% CrI [-3.08, 2.65])

124 or body mass (0.02 per g difference in body mass, $95 \%$ CrI [-0.10, 0.15]; Fig. S4), meaning

125 there is no evidence that the larger or faster bird sets the pace by leading. Interestingly, and in

126 contrast to the closely coordinated flight of birds flying in V-formation[12], there was no

127 correspondence between the front and back bird's median wingbeat frequency $(\beta=-0.23,95 \%$

128 CrI [-1.23, 0.75]; Fig. S3), indicating that their wingbeats cannot have been phase-locked

129 through most of the flight. All of these results were computed after accounting for the birds'

130 median airspeed, date of release and weather variables, none of which had an effect on

131 wingbeat frequency that was distinguishable from zero (Fig. S2, Table S1). Hence, whilst we

132 found no evidence in support of our hypothesis that birds of different sizes would specifically

133 have to adjust their wingbeat frequency or amplitude to stay together as a pair, we found clear

134 evidence that both birds in a pair increased their wingbeat frequency, independent of individual

135 size or solo flight speed.

137 In addition to these results for all releases, one similar-sized pair and one different-sized pair 138 separated during three releases each, which means we can fortuitously compare sections of 
139 paired and solo flight within the same release. The results for these six releases clearly confirm

140 that wingbeat frequency increases as a direct result of flying in a pair, because the birds' median

141 wingbeat frequency decreased by $1.01 \pm 0.30 \mathrm{~Hz}$ (mean \pm s.d.) after they separated and flew

142 solo (raw values with no covariates; Fig. 2E-H).

144 As previous research has shown that pigeons increase their wingbeat frequency by up to 0.1

$145 \mathrm{~Hz}$ as flock density increases [14], we analysed the effect of horizontal inter-individual distance

146 ranging from $0 \mathrm{~m}$ (i.e. directly above or below another bird) to $50 \mathrm{~m}$ (i.e. the cut off point for

147 flying in a pair) in a random sample of 100 wingbeats from each flight. This subsampling was

148 necessary due to the computational demands of dealing with the otherwise extremely large

149 volume of data. In total, we analysed 45,500 wingbeats from solo and paired flights. The results

150 demonstrate that birds flying with no horizontal spacing did indeed have the highest wingbeat

151 frequency (increase of $1.21 \mathrm{~Hz}$ relative to flying solo; 95\% $\mathrm{CrI}[0.81,1.61], 21.6 \%$ ), with

152 wingbeat frequency decreasing by $0.011 \mathrm{~Hz}$ for every metre increase in horizontal spacing

153 (95\% CrI $[-0.012,-0.009])$. Thus, birds flying $50 \mathrm{~m}$ apart had an expected wingbeat frequency

$154 \quad 0.54 \mathrm{~Hz}$ lower than birds flying $0 \mathrm{~m}$ apart. Nevertheless, the act of flying in a pair still had a

155 larger overall effect than the distance between (or density of) the birds, which meant that even

156 birds flying $50 \mathrm{~m}$ apart increased their wingbeat frequency by $0.66 \mathrm{~Hz}(11.9 \%)$ relative to

157 flying solo. On average, birds flew with a median spacing $12.12 \pm 4.76$ metres (mean of the means for all pairs \pm standard deviation), which equates to a $1.07 \mathrm{~Hz}$ increase in wingbeat

159 frequency under the fitted relationship ( $c f$. the $1.00 \mathrm{~Hz}$ increase in median wingbeat frequency

160 was over the whole flight).

162 To explore the mechanism underlying this change in wingbeat frequency, we divided each 163 wingbeat into an upstroke and a downstroke phase. We defined these phases with respect to 
164 the peaks and troughs of the DB acceleration, which results from a combination of aerodynamic

165 and inertial forcing (see supplementary text for further detail). Whereas the dorsal aerodynamic

166 force is expected to peak mid-downstroke when the wing reaches its maximum flapping speed,

167 the dorsal inertial force is expected to peak at the start of the downstroke when the wing's

168 downwards acceleration is maximal. It follows that the maximum DB acceleration will be

169 reached somewhere between the start and middle of the kinematic downstroke, and similarly

170 for the minimum, which will be reached somewhere between the start and middle of the

171 kinematic upstroke. Hence, the downstroke phase, which we define as running from the point

172 of maximum to minimum DB acceleration, is expected to lag the kinematic downstroke slightly

173 (and similarly for the upstroke), but by less than a quarter of a cycle. With these definitions,

174 we found that birds reduced the median duration of the upstroke phase by $20.6 \%(-27.99$

175 milliseconds, 95\% CrI $[-35.61,-19.92])$ when flying in pairs, whereas the median duration of

176 the downstroke phase did not vary significantly (-3.53 milliseconds, 95\% CrI [-8.35, 1.38]; 2c-

177 d). It is clear by inspection of the wingbeat acceleration traces that this decrease in upstroke

178 duration results in a less asymmetric pattern of forces between the two wingbeat phases

179 (compare red versus blue lines in Fig. 1B-E), so that this change in wingbeat frequency essentially represents a switching of kinematic - if not aerodynamic - gait [24].

182 We hypothesise that a potential function of increasing wingbeat frequency and decreasing 183 oscillatory displacement of the body may be to enhance visual stability when attending to 184 nearby conspecifics. We therefore conducted a second experiment using head-mounted 185 accelerometers on six homing pigeons on short-range flights (950 m), flying solo and in pairs, 186 to determine if the same measured changes in wingbeat characteristics result in increased head 187 stability (see Methods). In close agreement with the first experiment, birds flying in pairs 188 increased their median wingbeat frequency by a mean of $1.10 \mathrm{~Hz} \pm 0.26$ relative to flying solo 
$189(6.6 \pm 0.42 \mathrm{~Hz}$ mean \pm s.d. for pairs; $5.5 \pm 0.46 \mathrm{~Hz}$ for solo). More importantly, however, the

190 results also show that the median peak-to-peak head displacement simultaneously decreased

191 by $5.3 \times 10^{-3} \mathrm{~m} \pm 6.6 \times 10^{-4}$ between solo and paired flight, representing a $30 \%$ reduction in the

192 amplitude of oscillatory head displacement (Fig. 3). This substantial improvement in

193 translational head stability is expected to result in a significant reduction in the retinal slip of

194 nearby objects including flight partners.

195

196 In summary, by reducing the duration of their upstroke phase, birds flying in a pair were able

197 to accommodate one additional wingbeat per second, whilst maintaining the same peak-to-peak

198 DB acceleration and simultaneously increasing the vertical stability of the head. Intuitively, a

199 higher-frequency kinematic gait adopted in paired flight will therefore be associated with a

200 higher mechanical power input than the lower-frequency flight kinematic gait adopted in solo

201 flight. Of course, a higher mechanical power requirement in paired flight could still be

202 associated with a lower cost of transport if this increased frequency were more than

203 compensated by an increased flight speed. However, whereas birds migrating in V-formations

204 are known to increase their airspeed as flock size increases [25], the birds in our study only

205 increased their airspeed by $3.3 \%$ when flying in pairs $\left(0.64 \mathrm{~m} \mathrm{~s}^{-1}\right.$ increase, $\left.95 \% \mathrm{CrI}[0.08,1.2]\right)$.

206 As we now explain, this increase in airspeed is much smaller than could have been expected to

207 be caused by the increase in wingbeat frequency alone, suggesting that there must have been

208 other compensatory changes in the kinematics.

209

210 In cruising flight, the net thrust of the wings balances the drag on the body, which scales as

$211 \rho U^{2} S_{b}$ where $\rho$ is air density and $S_{b}$ is body frontal area. For a $3 \%$ increase in airspeed $U$, it

212 follows that the time-averaged thrust can only have increased by just over $6 \%$. In contrast, the

213 thrust on a flapping wing has been shown to scale as $T \sim \rho S_{w} f^{2} A^{2}$, where $S_{w}$ is wing area, $f$ is 
214 wingbeat frequency, and $A$ is wingbeat amplitude [26]. Assuming all other things are equal, an

$21518 \%$ increase in wingbeat frequency would therefore be expected to produce about a $39 \%$

216 increase in thrust. However, we also know that the time-averaged lift must balance the bird's

217 weight when cruising, and that lift scales as $L \sim \rho S_{w} U^{2}$ in fast forward flight when the

218 contribution of the wing's own flapping speed can be ignored. This implies that the 3\% increase

219 in airspeed (i.e $6 \%$ increase in $U^{2}$ ) would have to have been countered by either a $6 \%$ decrease

220 in wing area, or an equivalent decrease in the proportionality constant of the scaling

221 relationship (i.e. the wing lift coefficient). Either kinematic change would be expected to

222 attenuate the thrust similarly, thereby reducing its expected increase to approximately $31 \%$.

223 This is still significantly higher than the $6 \%$ increase in thrust estimated on the basis of the $3 \%$

224 increase in airspeed, but the scaling $T \sim \rho S_{w} f^{2} A^{2}$ implies that these figures could be brought

225 into line by an accompanying 10\% decrease in wingbeat amplitude (see supplementary text for

226 further confirmation).

228 The aerodynamic power requirement of a flapping wing has been shown to scale as

$229 P_{A} \sim \rho c S_{w} f^{3} A^{2}$ where $c$ is the wing chord [26]. The inertial power requirement also varies in 230 proportion to $f^{3} A^{2}$, albeit with some further complications related to the effect of varying wing 231 span, but is an order of magnitude smaller than the aerodynamic power requirement $[27,28]$ so

232 is neglected here for simplicity. Assuming that the $18 \%$ increase in wingbeat frequency $f$

233 between solo and paired flight was accompanied by a $6 \%$ decrease in wing area $S_{w}$ and by a

$23410 \%$ decrease in wingbeat amplitude $A$ as required to meet the equilibrium conditions above,

235 then the aerodynamic power requirement (in $\mathrm{J} \mathrm{s}^{-1}$ ) would have increased by approximately $25 \%$

236 when flying in a pair. Given the $3 \%$ increase in airspeed, it follows that the aerodynamic cost

237 of transport (in $\mathrm{J} \mathrm{m}^{-1}$ ) must also have increased by some $21 \%$. However, another key benefit

238 often ascribed to flying in flocks is the ability to pool navigational knowledge. This should 
improve homing route accuracy [7,8], which could offset the increased aerodynamic cost of

240 transport and increased aerodynamic power requirement by simultaneously decreasing the

241 distance and duration of the flight.

243 We calculated the birds' route accuracy flying solo and in pairs using a weighted mean cosine

244 of the angle between the birds' heading and destination. Flying in a pair resulted in a $6.9 \%$

245 increase in route accuracy relative to both the Phase 1 and Phase 4 solo releases $(0.06,95 \%$ CrI

246 [0.01, 0.10]; Fig. 1), and a 6.5\% decrease in route length (Table S2). This is consistent both

247 with theory[7,8] and with previous empirical studies $[5,6]$. Offsetting the $21 \%$ higher cost of

248 transport when flying in a pair against the $6.5 \%$ reduction in route length, we would expect a

249 net increase of approximately $14 \%$ in the total mechanical energy expended when flying home

250 to the loft in a pair. The increase in the total metabolic energy expended could be higher or

251 lower than this, depending upon whether and how the efficiency of the flight muscles varies

252 with flapping frequency, but it is reasonable to assume that an increase in mechanical energy

253 would also be associated with an increase in metabolic energy.

255 Whilst these figures are necessarily approximate, the qualitative conclusion of this analysis -

256 that the energy expended returning to the loft would have been higher in paired than in solo

257 flight - is robust to the uncertainty in our estimates of the changes in wingbeat frequency and

258 airspeed. Substituting the limits of the 95\% CrI's for these variables into the preceding 259 calculations leads to an estimated $4 \%$ or $24 \%$ increase in the total energy expended in paired

260 flight if the variables fall at their respective upper or lower limits, and an estimated $10 \%$ or

$26117 \%$ increase if they fall at opposite ends of their limits. These are worst-case figures for how

262 the uncertainties could combine, all of which lead to the conclusion that flying in pairs is

263 associated with a substantial net increase in energy consumption over the flight. As we now 
264 discuss, this surprising result indicates: that $(i)$ the act of flying in a pair necessitates birds to

265 alter their wing kinematics to a higher-frequency kinematic gait; and that either (ii) the

266 navigational benefits of flying in a pair are sufficient to outweigh the increased cost of transport

267 over longer homing distances, such that flying in pairs makes sense as a general homing

268 strategy, or (iii) there are other benefits of paired flight that outweigh its net energetic cost even

269 over short distances.

270

\section{Discussion}

273 One of the most commonly cited reasons for travelling as a group is to reduce energy expenditure and enhance locomotor performance $[11,12]$. Previous research in cluster flocking pigeons has shown that the energetic cost of flocking increases slightly with increasing flock density [14]. However, this study did not compare the cost of flying in a cluster flock relative to the alternative of flying solo, which means, as we now show, that previous work has

278 inadvertently understated the energetic costs of cluster flocking by an order of magnitude. Specifically, whereas pigeons have previously been shown to increase their wingbeat frequency by a mere $0.1 \mathrm{~Hz}$ with increasing flock density [14], our results show that the very act of flying with another bird increases a pigeon's wingbeat frequency by $1.0 \mathrm{~Hz}(18 \%)$, which results in an estimated $21 \%$ increase in the aerodynamic cost of transport. Although birds flying in pairs were simultaneously able to offset some of the energetic cost by flying more accurate routes home, the increases in route accuracy and airspeed were insufficient to compensate for the increased aerodynamic power requirements, which resulted in a net energetic loss on the order of $14 \%$ when flying moderate distances $(\sim 7 \mathrm{~km})$ together in a cluster formation.

287 Moreover, the fact that pigeons flying in pairs display a $18 \%$ increase in wingbeat frequency over solo flight suggests that the majority of the additional cost comes merely from the act of 
flying with another individual, rather than from the density of the flock, the relative spatial

290 position of the bird, or the size of its partner. Indeed, the size of a bird's partner, and whether

291 that bird was a leader or follower, had almost no effect on its measured wingbeat pattern. Even

292 so, differences in inter-individual horizontal spacing did result in a $0.54 \mathrm{~Hz}$ difference in

293 wingbeat frequency between birds travelling 0 to $50 \mathrm{~m}$ apart, with this increase ranging from

29411.9 to $21.6 \%$, respectively. Thus, the act of flying with a conspecific resulted in a substantial

295 alteration of the wingbeat - even the adoption of a different kinematic gait. As we now explain,

296 not only does this earlier omission mean that the costs of flocking have been massively

297 underestimated - it also means that their mechanistic origin must be re-evaluated.

299 Two key hypotheses have been proposed for the increase in wingbeat frequency seen in denser 300 cluster flocks: ( $i$ ) negative aerodynamic interactions between flock members and (ii) increased need for control and collision avoidance [14]. Whereas a focus on the small effects of spacing

302 within a flock led previous work to hypothesise a possible aerodynamic basis to the costs of 303 cluster-flocking [14], our work clearly demonstrates that both birds within a pair increase their 304 wingbeat frequency, which suggests that these effects are unlikely to have been related to negative aerodynamic flow interactions, since the bird in front does not fly in the wake of the bird behind. On the other hand, higher wingbeat frequencies can be used to enhance both stability and manoeuvrability [14,29-31]. We therefore hypothesise that the increase in

308 wingbeat frequency is related to paired flight necessitating a greater degree of control, which could come about in two different ways. First, flying with conspecifics may require enhanced manoeuvrability and control because birds need to adjust their orientation continuously and

311 rapidly, both to stay together and to avoid collisions [14]. Second, birds may require enhanced

312 visual stability when flocking, in order to observe and coordinate with individuals whose

313 proximity makes the effects of motion parallax significant [32,33]. 
315 Birds make kinematic control inputs on a wingbeat-to-wingbeat basis, so increasing wingbeat

316 frequency will increase the rate at which control inputs can be made, enhancing the bird's

317 ability to respond to the movements of others and increasing the precision of its response.

318 Moreover, increased wingbeat frequency is expected to amplify flight stability [31], which

319 could both enhance control and visual stability. Unlike humans, birds have a limited range of

320 eye movement and therefore visual stabilisation is facilitated by compensatory motion of the

321 sophisticated avian head-neck system and is mediated by visual, vestibular and proprioceptive

322 cues [34,35]. Without image stabilisation mechanisms, birds would have difficulty

323 differentiating the motion of a target or obstacle from head or body motions, which is especially

324 problematic when viewing nearby objects or conspecifics. Our results show that dorsal body

325 displacement in reaction to the wingbeat is attenuated by $23 \%$ in the higher-frequency

326 kinematic gait adopted in paired flight (see supplementary text for theoretical analysis), which

327 should naturally translate into a reduced amplitude of head motion. Indeed, using data from

328 head-mounted accelerometers, we prove that the heads of birds flying in pairs experience

329 significantly less vertical head displacement relative to flying solo $(\sim 30 \%)$. Pigeons flying in

330 pairs have also been recorded to reduce their angular head saccades relative to flying solo,

331 which suggests either an increased focus on their partner or a decreased focus on the

332 environment [36]. Furthermore, the linearly declining effect of paired flight with increasing

333 inter-individual distance suggests that the observed changes in wingbeat frequency are less to

334 do with the local aerodynamic environment, the effects of which drop off sharply, and are

335 instead related to the coordination of flock flight, noting that the effects of motion parallax

336 decline linearly with distance. Together, our results suggest that a higher wingbeat frequency

337 may be prerequisite for flocking flight because of the increased demands for stability and

338 control. 
340 Managing energy expenditure is critical for survival, and is a primary focus for natural

341 selection. While our results demonstrate that birds can derive navigational benefits from flying

342 in pairs even during short-range flights along familiar routes, the $7 \%$ increase in route accuracy

343 over this range was apparently insufficient to counterbalance the cost of the $18 \%$ increase in

344 wingbeat frequency, with an estimated increase in mechanical energy expenditure on the order

345 of $14 \%$. Despite this, only six releases had to be repeated due to birds separating at the start,

346 and only twelve out of 116 pair releases (10\%) resulted in separation part-way through the

347 release. Hence, the observed preference for paired flight suggests that either $(i)$ the general

348 strategy of flocking is adaptive because the navigational benefits of flocking are sufficient to

349 outweigh the increased cost of transport when homing over longer distances, or (ii) the other

350 benefits of flocking, such as predator protection, outweigh the increase in energy expenditure

351 required to fly in pairs, even over quite short distances.

353 Although minimizing energy consumption may not be an especially strong selection pressure

354 for homing pigeons that have been selectively bred to return quickly to the loft, and which have ad libitum access to feed, the results of our study nevertheless indicate that flying with conspecifics entails an energetically expensive alteration to wingbeat kinematics. As many other species of birds also preferentially fly in cluster flocks, our results suggest that the

358 additional benefits of flocking must outweigh any accompanying increase in energy expenditure. The overall $9 \%$ reduction in homing flight time that we observed represents a $9 \%$ reduction in the period over which our birds were exposed to predation risk when returning to

361 the loft. Moreover, not only does the act of flying in a pair dilute the chance of fatality during 362 a predation event by $50 \%$, but the probability that such a predation event is successful decreases 363 as flock size increases, presumably through a combination of increased opportunity for 
vigilance and predator confusion effects [37]. Therefore, for pigeons, the ultimate benefits of

365 flocking, such as protection from predators and the pooling of navigational knowledge, must

366 together outweigh the energetic cost of flying with conspecifics.

368 Over longer flight distances or circumstances where an individual has substantially less navigational knowledge than the flock, it is possible that the navigational benefits of flocking might be sufficient to produce a net reduction in the amount of energy expended despite the increased aerodynamic power requirement that we report. In order to gain energetic savings

372 from a $21 \%$ increase in the cost of transport $\left(\mathrm{J} \mathrm{m}^{-1}\right)$, the homing distance $(\mathrm{m})$ would have to be

$373>17 \%$ shorter when flying in a pair to result in net energetic savings $(\mathrm{J})$. In this scenario, birds would experience an increased rate of energy expenditure which would be compensated by net energetic savings and a reduced risk of predation. Either way, the birds in our study still opted to fly in pairs despite collective travel resulting in an energetic loss at the individual level.

Overall, the results of our study of a cluster flocking species stand in contrast to previous studies of birds flying in V-formations [11-13]. Formation flight is typically utilised by medium to large sized birds during goal-orientated movement, whereas cluster flock formations are utilised by smaller birds, such as starlings, in movement ranging from orientated to highly tortuous motion $[15,16]$. Bird size and the complexity of movement paths may both contribute to the observed differences in wingbeat patterns between flock formation types.

384 Whilst birds flying in V-formations are able to fly in aerodynamically optimal positions to conserve energy, the naturally higher wingbeat frequencies of smaller birds, their smaller turning angles, and the rapidity of their directional changes may preclude flying in energysaving formations and instead necessitate a wingbeat pattern that facilitates a greater degree of control. Thus, the demands of moving in irregular three-dimensional flocks may alter the way 
in which a bird flies. Overall, our results provide key new insights into both the biomechanical

391 access to collective navigational knowledge and predator protection. Taken together, our

392 results demonstrate that flocking is, for pigeons, both fundamentally important and

393 fundamentally expensive.

Materials and Methods

396

a) Experiment 1

401 Twenty homing pigeons aged 1 or 3 years were used. Body size was quantified by measuring tarsus length (mm) and body mass (g). Tarsus length was measured with callipers sensitive to $0.1 \mathrm{~mm}$ using the methods described in Sutherland et al. [22]. Body mass was measured using digital scales (Salter ARC Electronic Kitchen Scales, Salter, UK; \pm 1 g). All subjects completed the start of the experiment. The subjects were housed with $\sim 120$ other pigeons in two neighbouring lofts at the Oxford University Field Station, Wytham, UK (5146'58.2”N, $\left.1^{\circ} 19^{\prime} 2.7^{\prime \prime} \mathrm{W}\right)$. Access to water, grit and a standard pigeon feed mix were available ad libitum at all times in the loft. The protocols outlined in this paper were approved by the Local Ethical

410 Review Committee of the University of Oxford's Department of Zoology. 
414 The birds were tracked using 5 Hz GPS loggers (QStarz BT-Q1300ST, 15 g) and 200 Hz tri-

415 axial accelerometers ( $\pm 16 \mathrm{~g}$; Axivity AX3, $11 \mathrm{~g}$ ), which were attached via Velcro strips glued

416 to trimmed feathers on the birds' back. In total, the loggers and fastenings weighed $27 \mathrm{~g}$. To

417 enable subjects to adapt to carrying the additional mass, clay weights were attached to them

418 throughout the pre-training and experimental periods, which meant the weights were attached

419 for a minimum of 43 days prior to the start of the experiment. The weights were exchanged for

420 the loggers immediately prior to each release. GPS and accelerometer data were downloaded

421 using QTravel (Qstarz International Co., Ltd., Taipei, Taiwan; version 1.48(T)) and Open

422 Movement (Om) GUI Application (Newcastle University, UK; version 1.0.0.28), respectively.

423

424 The weather, including mean wind speed per minute $\left(\mathrm{ms}^{-1}\right)$, a running mean of the wind bearing over the previous ten minutes, temperature $\left({ }^{\circ} \mathrm{C}\right)$, humidity $(\%)$ and barometric pressure $(\mathrm{hPa})$,

were recorded using a WS2083 Professional Wireless Weather Station with USB upload

427 (Aercus Instruments, UK) situated $5.5 \mathrm{~m}$ above the ground near the pigeon lofts and Cumulus

428 Weather Station Software (Sandaysoft; version 1.9.4).

iii) Experimental procedures

432 The release site was located $7.06 \mathrm{~km}$ from the loft on a bearing of $282^{\circ}$ (Barnard Gate; $\left.43351^{\circ} 47^{\prime} 48.1^{\prime \prime} \mathrm{N}, 1^{\circ} 25^{\prime} 3.3^{\prime \prime} \mathrm{W}\right)$. The experiment consisted of four phases: Phase 1 - six individual 434 releases (solo 1); Phase 2 - six releases with a bird of a similar size (similar-sized pair); Phase 4353 - six releases with a bird of a different size (different-sized pair); and Phase 4 - six individual 436 releases (solo 2). Bird pairings can be found in Table S3. Releases were conducted between 437 June and September 2015, on days when the sun was visible and the wind speed was $<7 \mathrm{~ms}^{-1}$. 438 Subjects participated in a maximum of two releases per day, with a minimum of three hours 
between each release. The birds had to complete a minimum of one third of the flight together

440 for the flight to be included in the analysis. If the birds spent less than one third of the flight

441 together, the flight was repeated. In total, six releases out of 116 pair releases had to be

442 repeated. One different-sized pair did not complete the final pair release after repeatedly

443 separating. The Velcro failed on bird S27 after the third release with a different-sized bird

444 therefore the pairing S27 and S84 only completed three different size pair releases and S27 did

445 not complete the final solos. In addition, S13 only completed one final solo before the Velcro

446 failed, S87 completed four final solos, and S05 and S25 completed five final solos each. The

447 remaining 15 birds all completed the final solo releases.

iv) Data processing

451 Data were processed using the procedures outlined in Taylor et al. [20]. For each GPS point, 452 the orthodromic (great-circular) distance travelled and birds' final bearing from the previous 453 point were calculated using the haversine formula and forward azimuth, respectively. The dorsal accelerometer measurements were filtered by taking a running mean over three data points $(0.015 \mathrm{~s})$. Static acceleration (or gravity) was removed by subtracting a running mean over 15 wingbeat cycles ( $>2 \mathrm{~s}$; Fig S5). The wingbeat frequency (number of wingbeats per second; $\mathrm{Hz}$ ) and peak-to-peak dorsal body (DB) acceleration $(g)$ using the dorsal acceleration signal (Z-axis) were calculated for each individual wingbeat. The amplitude of the DB displacement $(\mathrm{mm})$ was then calculated by the double integration of dorsal accelerometer measurements $[14,20]$. In addition, we calculated the duration of the "downstroke" from the

461 peak downstroke force (maximum $g$-force) to the lower reversal point (minimum $g$-force). The

462 "upstroke phase" duration, which included the start of the downstroke, was measured from

463 minimum $g$-force to the maximum. We used the maximum and minimum $g$-force peaks to 
divide the wingbeat for consistency, as the start of the kinematic downstroke was not distinguishable in the data from paired flights. See supplementary text for further analysis.

467 Wind support, crosswind and airspeed were calculated using the methods described in Safi et al.[38] using the measurements from the weather station and speed derived from the GPS devices. Humid air density $\left(\mathrm{kg} \mathrm{m}^{-3} ; \rho_{\text {air }}\right)$ was calculated from measures of barometric pressure $(\mathrm{hPa} ; P)$, temperature $\left({ }^{\circ} \mathrm{C} ; T_{C}\right)$ and relative humidity $(\% ; \phi)$ recorded by the weather station, using the following calculation derived from the ideal gas law:

$$
\rho_{\text {air }}=\left(\frac{P_{d}}{R_{d} T}\right)+\left(\frac{P_{v}}{R_{v} T}\right)
$$

where $P_{d}$ is pressure of dry air $(\mathrm{Pa}), R_{d}$ is gas constant for dry air $[287.05 \mathrm{~J} /(\mathrm{kg} * \mathrm{~K})], P_{v}$ is pressure of water vapour $(\mathrm{Pa}), R_{v}$ is gas constant for water vapour $[461.495 \mathrm{~J} /(\mathrm{kg} * \mathrm{~K})]$ and $T$ is ambient temperature $(\mathrm{K}) . P_{v}$ can be calculated from the saturation of vapour pressure $\left(P_{\text {sat }}\right)$ and relative humidity $(\phi)$ :

$$
P_{v}=\phi P_{s a t}
$$

We used the Arden-Buck [39,40] equation to calculate $P_{\text {sat }}$, where $P_{\text {sat }}(\mathrm{hPa})$ is calculated as:

$$
P_{\text {sat }}=0.61121 \exp \left(\left(18.678-\frac{T_{C}}{234.5}\right)\left(\frac{T_{C}}{257.14+T_{C}}\right)\right)
$$

$P_{d}$ can then be calculated from the barometric pressure $(P)$ and the vapour pressure of water $\left(P_{v}\right)$

$$
P_{d}=\mathrm{P}-\mathrm{P}_{v}
$$

484 The birds' route accuracy was calculated using a weighted mean cosine of the angle $(\theta)$ 485 between the birds bearing and the bearing to the loft for each timestep, where $\theta$ is equal to the smallest angle difference so that $\theta$ ranged from 0 (heading directly to the loft) and 180 (heading 
directly away from the loft), and the orthodromic distance between each GPS point $(d)$ using the following calculation:

$$
\frac{1}{D} \sum_{i=1}^{n} d_{i} \cos \theta_{i}
$$

where $D$ is the total distance flown. Route accuracy is, therefore, on a scale of -1 (heading in a [41], but enables us to calculate the accuracy for sections of a flight, rather than a whole flight, which is necessary if the birds separate during the flight and fly solo.

The data were trimmed within a $200 \mathrm{~m}$ radius around the release site and the loft to remove take-off and landing. When analysing the pair tracks, sections of flight where the birds were $\geq$ $50 \mathrm{~m}$ apart was excluded. In addition, if the birds swapped front-vs-back positions, the bird who spent the majority of the flight in front based on GPS positioning was identified and the rest of the data from when the other bird was in front was excluded.

505 We analysed the data using Bayesian hierarchical models, which are analogous to mixed models in frequentist methods and enabled us to account for the effects of each bird both as an $(i, j)$ was assumed to be normally distributed,

$$
\bar{W}_{i, j} \sim N\left(\delta_{0} 1_{\text {pair }}+\delta_{1}\left\|T_{i}-T_{j}\right\|+\omega_{i} \mu_{i}+\left(1-\omega_{i}\right) \mu_{j}+\gamma X, \sigma\right)
$$


512 where $1_{\text {pair }}$ is an indicator variable equal to 1 if the bird flew in a pair, and 0 for solo flights.

$513 \delta_{0}$ is the difference in wingbeat frequency between the solo and paired flight. $\delta_{1}$ is the

514 difference in wingbeat frequency for every $\mathrm{mm}$ absolute difference in tarsus length $(T)$ between

515 the pair $(i, j)$. For solo flights, the term involving $\delta_{1}$ equals zero. The expression $\omega_{i} \mu_{i}+(1-$

$\left.516 \omega_{i}\right) \mu_{j}$ represents a weighted average of the solo wingbeat frequency $(\mu)$ of birds $i$ and $j$ with a

517 mixing weighting $\left(\omega_{i}\right)$, which determines the weight placed on the bird's own solo wingbeat

518 frequency $\left(\mu_{i}\right)$ relative to that of its partner $\left(\mu_{j}\right)$. For solo flights, this weighted average equals

519 bird $i$ 's solo wingbeat frequency $\left(\mu_{i}\right)$. The weighting is bounded to lie between 0 and 1 and was determined by a logistic sigmoid function of the absolute difference in tarsus length,

$$
\omega_{i}=\operatorname{logit}^{-1}\left(\eta_{1}+\eta\left\|T_{i}-T_{j}\right\|\right)
$$

522 However, across all cases, there was no consistent effect of tarsus difference on the mixing weighting.

525 Finally, $\gamma X$ represents the effect of the covariates, which accounts for median wind support (m

$\left.526 \mathrm{~s}^{-1}\right)$, median crosswind $\left(\mathrm{m} \mathrm{s}^{-1}\right)$, median temperature $\left({ }^{\circ} \mathrm{C}\right)$, median humidity $(\%)$, humid air 527 density $\left(\mathrm{kg} \mathrm{m}^{-3}\right)$ and the date of release treated as a categorical variable. The birds' median 528 airspeed $\left(\mathrm{m} \mathrm{s}^{-1}\right)$ was also added as a covariate on all models except for models of airspeed. We used airspeed rather than ground speed as a covariate because ground speed and wind support 530 were correlated (Fig. S5). In terms of the response variable, there was almost no difference

531 between the models of airspeed and ground speed as the model accounts for the effect of wind $532(0.64,95 \% \mathrm{CrI}[0.08,1.20]$ compared to $0.69,95 \% \mathrm{CrI}[0.10,1.28])$. For consistency with the covariates, we present the results of the model for airspeed. A covariate indicating whether the

534 bird was a leader or follower was also added to the model in a secondary analysis to determine 535 the effect of the birds' position on wingbeat frequency. 
537 In addition to modelling the median values, we also took a random sample of 100 individual

538 wingbeats to analyse the effect of horizontal distance between birds in pairs. We analysed

539 horizontal distance rather than three-dimensional distance because GPS precision is generally

540 poorer in the vertical than the horizontal [42]. Horizontal distance (m) was added as a covariate

541 to the paired data, along with a categorical covariate identifying the specific bird and flight to

542 account for the repeated measures of 50 wingbeats from one flight. In total, 44,500 wingbeats

543 from 454 unique bird and flight combinations were analysed.

545 To investigate whether the birds were flying in phase, we used the following model to identify

546 whether the median wingbeat frequency of the follower $\left(\bar{W}_{F}\right)$ is related to the leader $\left(\bar{W}_{L}\right)$ in

547 pair $(P)$ :

$$
\bar{W}_{F} \sim N\left(\alpha_{P}+\beta_{P} \bar{W}_{L}+\gamma X, \sigma^{\prime}\right)
$$

550 We also investigated whether the difference between the leader and follower's tarsus length, body mass or solo airspeed $(S)$ predicted why the bird was a leader $(L)$ in the pair using a

552 Bernoulli regression:

$$
L_{i} \sim \operatorname{Bernoulli}\left(\alpha_{P}+\beta_{P} S_{i}\right)
$$

555 The model priors were centred on the null hypothesis using the mean, standard deviation and square root standard deviation of the solo data (Table S4). Eight Markov chain Monte Carlo

557 (MCMC) chains were run simultaneously, each with 12,500 warm-up and 12,500 model 558 iterations, which resulted in 100,000 samples for each posterior distribution. For the model 559 involving raw wingbeat data, the model was run for 10,000 samples due to the size of the model. Across all estimated models and parameters, we detected convergence in the sampling 
561 distribution as determined by using a criterion $\hat{R} \leq 1.1$ on all parameters[43]. The number of

562 divergent iterations was $0.0-1.3 \%$ of the total sample size. The code for the wingbeat frequency

563 model and the model output can be found in the Supplementary Materials.

564

565 In addition to these results, one similar-sized pair (birds B01 and B82 with a tarsus length of

$566 \quad 32.4 \mathrm{~mm}$ and $33.3 \mathrm{~mm}$, respectively) and one different-sized pair (birds B01 and B07 with a

567 tarsus length of $32.4 \mathrm{~mm}$ and $35.2 \mathrm{~mm}$, respectively) split and flew solo for more than $30 \%$ of

568 the flight for three of their six releases. As the sample size is low, only descriptive statistics

569 can be performed comparing the paired ( $<50 \mathrm{~m}$ distance between birds) and solo flight $(>300$

$570 \mathrm{~m}$ distance).

571

572 We approximated the number of wingbeats difference between the solo and paired flight using

573 the total flight distance (excluding the 200m take-off and landing) and the model results from

574 route accuracy, airspeed and wingbeat frequency (Table S2). We calculated the number of wingbeats difference as not all of birds completed $100 \%$ of the paired flights together or with one leader.

Data processing and analysis were conducted using MATLAB (MathWorks, Natick, USA; version R2015a) and the open-source software R (version 3.4.2) [44]. Bayesian models were written in Stan [45] using the R interface RStan (version 2.16.2) [46]. 
586 Six homing pigeons aged 3 to 10 years old were used. The pigeons were held under the same

587 conditions as outlined above and all had experience of experimental releases and the release

588 site. The protocols outlined in this paper were approved by the University of Oxford's Zoology

589 Animal Welfare Ethical Review Board (No. APA/1/5/ZOO/NASPA/Biro/

590 PigeonsHeadmountedsensors).

591

592

ii) Data logging

593

594 We used a custom-built 'p-Sensor' to simultaneously record head movement and position. The

595 p-Sensor included an IMU with a combination of a tri-axial gyroscope, tri-axial accelerometer

596 and tri-axial magnetometer recording at $60 \mathrm{~Hz}$, and a GPS logger recording at $10 \mathrm{~Hz}$. The IMU

597 was mounted using double-sided tape onto a custom-made and custom-fitted wire mask

598 designed to fit each bird's head. The GPS logger, SD card, battery and microcomputer were

599 placed in an elasticated backpack on the birds back. The instrumentation, mask, and backpack

600 weighed $28.1 \mathrm{~g}$ and constituted $4.9 \%$ of the body mass of the smallest bird, of which the IMU

601 unit on the bird's head only weighed $1 \mathrm{~g}$. For more details, see Kano et al. [36].

602

603 All birds were habituated to wearing the custom-made mask for at least seven days prior to the

604 flight. For each day of habituation, the bird was fitted with a mask and carefully monitored for

605 two hours within its home loft for signs of discomfort and abnormal patterns of locomotion.

606 After seven days of habitation in the loft, the pigeons were released outside the loft and allowed

607 to fly freely under close observation.

608

$609 \quad$ iii) Experimental procedures

610 
611 The release site was located $0.95 \mathrm{~km}$ from the loft on a bearing of $199^{\circ}$ (Wytham Woods;

$\left.61251^{\circ} 46^{\prime} 29.4^{\prime} \mathrm{N}, 1^{\circ} 19^{\prime} 18.7^{\prime \prime} \mathrm{W}\right)$. The experiments were conducted on the $23^{\text {rd }}$ July 2017.

613 Releases were only conducted when the wind was low $\left(<5 \mathrm{~m} \mathrm{~s}^{-1}\right)$ and the sun's disc was visible.

614 For the day of testing, the birds were fitted with a mask and allowed to habituate to wearing

615 the mask in the home loft before being transported to the release site by car. The birds were

616 released once solo and once in a pair on the same day. The release order was randomised.

617

iv) Data processing and analysis

621 The data processing was conducted as outlined in Taylor et al. [20]. Vertical (Z-axis) accelerometer measurements were smoothed by taking a running mean over five datapoints $(0.083 \mathrm{~s})$ and then filtered using a $4^{\text {th }}$ order high-pass Butterworth filter with a cut-off frequency of $1 \mathrm{~Hz}$. The peak-to-peak vertical head displacement was calculated by the double integration 625 of the vertical accelerometer measurements. We compared the median peak-to-peak vertical 626 head displacement between solo and paired releases for each bird.

628 The raw data will be available on data dryad after acceptance.

630 Acknowledgments:

631 We thank Lucy Larkman, Dave Wilson and Phil Smith for animal husbandry and technical 632 support and Fumihiro Kano for his contributions to the development of the head-mounted 633 sensor and mask for head attachment. We thank Marco Klein Heerenbrink, Oliver Padget, Jay 634 Willis and Simon Taylor for helpful discussions. We thank Eoin Malins from the University 635 of Oxford's Doctoral Training Centre for providing computer access. L.A.T. and J.A.W. were 
636 funded by the Biotechnology and Biological Sciences Research Council (BBSRC) UK (grant

637 number BB/J014427/1). B.L. was funded by Engineering and Physical Sciences Research

638 Council (EPSRC) UK (grant number EP/F500394/1).

639

$640 \quad$ References

641

642 1. Krause J, Ruxton GD. Living in groups. Oxford, UK: Oxford University Press; 2002.

643 2. Herbert-Read JE. Understanding how animal groups achieve coordinated movement. J

$644 \quad$ Exp Biol. 2016;219: 2971-2983. doi:10.1242/jeb.129411

645 3. Vicsek T, Zafeiris A. Collective motion. Phys Rep. Elsevier B.V.; 2012;517: 71-140. doi:10.1016/j.physrep.2012.03.004

647 4. Ward AJW, Herbert-Read JE, Sumpter DJT, Krause J. Fast and accurate decisions through collective vigilance in fish shoals. Proc Natl Acad Sci U S A. 2011;108: 23122315. doi:10.1073/pnas.1101616108

650 5. Biro D, Sumpter DJT, Meade J, Guilford T. From Compromise to Leadership in Pigeon 651 Homing. Curr Biol. 2006;16: 2123-2128. doi:10.1016/j.cub.2006.08.087

652 6. Dell'Ariccia G, Dell'Omo G, Wolfer DP, Lipp HP. Flock flying improves pigeons' homing: GPS track analysis of individual flyers versus small groups. Anim Behav. 2008;76: 1165-1172. doi:10.1016/j.anbehav.2008.05.022

655 7. Simons AM. Many wrongs: the advantage of goup navigation. Trends Ecol Evol. 2004;19: 453-455. doi:10.1016/j.tree.2004.07.001

657 8. Berdahl A, Torney CJ, Ioannou CC, Faria JJ, Couzin ID. Emergent Sensing of Complex Environments by Mobile Animal Groups. Science. 2013;339: 574-576. doi:10.1126/science. 1225883

660 9. Bill RG, Herrnkind WF. Drag Reduction by Formation Movement in Spiny Lobsters. 
Science. 1976;193: 1146-1148.

662 10. Liao JC, Beal DN, Lauder G V, Triantafyllou MS. Fish Exploiting Vortices Decrease Muscle Activity. Science. 2003;302: 1566-1569. doi:10.1126/science.1088295

664 11. Weimerskirch H, Martin J, Clerquin Y, Alexandre P, Jiraskova S. Energy saving in flight formation. Nature. 2001;413: 697-698. doi:10.1038/35099670

666 12. Portugal SJ, Hubel TY, Fritz J, Heese S, Trobe D, Voelkl B, et al. Upwash exploitation Publishing Group; 2014;505: 399-402. doi:10.1038/nature12939

13. Voelkl B, Portugal SJ, Unsöld M, Usherwood JR, Wilson AM, Fritz J. Matching times of leading and following suggest cooperation through direct reciprocity during Vformation flight in ibis. Proc Natl Acad Sci U S A. 2015;112: 2115-20. doi:10.1073/pnas.1413589112

673 14. Usherwood JR, Stavrou M, Lowe JC, Roskilly K, Wilson AM. Flying in a flock comes at a cost in pigeons. Nature. Nature Publishing Group; 2011;474: 494-497. doi:10.1038/nature10164

15. Bajec IL, Heppner FH. Organized flight in birds. Anim Behav. Elsevier Ltd; 2009;78: 777-789. doi:10.1016/j.anbehav.2009.07.007

16. Heppner FH. Avian flight formations. Bird-Banding. 1974;45: 160-169.

679 17. Schmidt-Nielsen K. Locomotion: Energy Cost of Swimming, Flying, and Running. Science. 1972;177: 222-228. doi:10.1126/science.177.4045.222

681 18. Tobalske BW, Hedrick TL, Dial KP, Biewener AA. Comparative power curves in bird 682 flight. Nature. 2003;421: 363-366. doi:10.1038/nature01367.1.

683 19. Nagy M, Akos Z, Biro D, Vicsek T, Ákos Z, Biro D, et al. Hierarchical group dynamics 684 in pigeon flocks. Nature. 2010;464: 890-893. doi:10.1038/nature08891

685 20. Taylor LA, Portugal SJ, Biro D. Homing pigeons (Columba livia) modulate wingbeat 
characteristics as a function of route familiarity. J Exp Biol. 2017;220: 2908-2915.

687

21. Tobalske BW, Dial KP. Flight kinematics of black-billed magpies and pigeons over a wide range of speeds. J Exp Biol. 1996;199: 263-280. Available: http://www.ncbi.nlm.nih.gov/pubmed/9317775

22. Sutherland WJ, Newton I, Green R. Bird ecology and conservation: a handbook of techniques. Oxford, UK: Oxford University Press; 2004.

23. Esri, HERE, DeLorme, Intermap, INCREMENT P, GEBCO, et al. World Topographic Map. [Internet]. 2013 [cited 21 Jun 2017]. Available: http://services.arcgisonline.com/ArcGIS/rest/services/World_Topo_Map/MapServer

24. Tobalske BW. Biomechanics and Physiology of Gait Selection in Flying Birds. Physiol Biochem Zool. 2000;73: 736-750. doi:10.1086/318107

25. Hedenström A, Åkesson S. Flight speed adjustment by three wader species in relation to winds and flock size. Anim Behav. 2017;134: 209-215. doi:10.1016/j.anbehav.2017.10.022

26. Floryan D, Van Buren T, Smits AJ. Efficient cruising for swimming and flying animals is dictated by fluid drag. PNAS. 2018; 201805941. doi:10.1073/pnas.1805941115

27. van den Berg C, Rayner J. The moment of inertia of bird wings and the inertial power requirement for flapping flight. J Exp Biol. 1995;198: 1655-64. Available: http://www.ncbi.nlm.nih.gov/pubmed/9319563

28. Burgess SC, Lock RJ, Wang J, Sattler GD, Oliver JD. The effect of aerodynamic braking on the inertial power requirement of flapping flight: case study of a gull. Int J Micro Air Veh. 2014;6: 117-128. doi:10.1260/1756-8293.6.2.117

29. Hedrick TL, Cheng B, Deng X. Wingbeat Time and the Scaling of Passive Rotational Damping in Flapping Flight. Science. 2009;324: 252-255. doi:10.1126/science. 1168431 
711

712

713

714

715

716

717

718

719

720

721

722

723

724

725

726

727

728

729

730

731

732

733

734

735

30. Williams CD, Biewener AA. Pigeons trade efficiency for stability in response to level of challenge during confined flight. Proc Natl Acad Sci U S A. 2015;112: 3392-3396. doi:10.1073/pnas. 1407298112

31. Taylor GK, Thomas ALR. Animal flight dynamics II. Longitudinal stability in flapping flight. J Theor Biol. 2002;214: 351-370. doi:10.1006/jtbi.2001.2470

32. Frost BJ, Wylie DR, Wang YC. The processing of object and self-motion in the tectofugal and accessory optic pathways of birds. Vision Res. 1990;30: 1677-1688. doi:10.1016/0042-6989(90)90152-B

33. Frost J. The optokinetic basis of head-bobbing in the pigeon. J Exp Biol. 1978;74: 187195.

34. Gioanni H. Stabilizing gaze reflexes in the pigeon (Columba livia) - I. Horizontal and vertical optokinetic eye $(\mathrm{OKN})$ and head (OCR) reflexes. Exp Brain Res. SpringerVerlag; 1988;69: 567-582. doi:10.1007/BF00247310

35. Land MF. The roles of head movements in the search and capture strategy of a tern (Aves, Laridae). J Comp Physiol - A Sensory, Neural, Behav Physiol. 1999;184: 265272. doi:10.1007/s003590050324

36. Kano F, Walker J, Takao S, Biro D. Head-mounted sensors reveal visual attention of free-flying homing pigeons. J Exp Biol. 2018;221. doi:10.1098/rstb.2011.0034

37. Kenward RE. Hawks and Doves: Factors Affecting Success and Selection in Goshawk Attacks on Woodpigeons. J Anim Ecol. 1978;47: 449. doi:10.2307/3793

38. Safi K, Kranstauber B, Weinzierl R, Griffin L, Rees EC, Cabot D, et al. Flying with the wind: scale dependency of speed and direction measurements in modelling wind support in avian flight. Mov Ecol. 2013;1: 4. doi:10.1186/2051-3933-1-4

39. Buck AL. New equations for computing vapor pressure and enhancement factor. J Appl Meterology. 1981;20: 1527-1532. 
40. Buck AL. Buck Research CR-1A User's Manual. Boulder, CO, USA: Buck Research Instruments; 1996.

41. Batschelet E. Circular statistics in biology. London, UK: Academic Press; 1981.

42. Acharaya R. Understanding satellite navigation. London, UK: Academic Press; 2014.

43. Gelman A, Rubin DB. Inference from iterative simulation using multiple sequences. Stat Sci. 1992; 7: 457-472.

44. R Core Team. R: A language and environment for statistical computing [Internet]. Vienna, Austria: R Foundation for Statistical Computing; 2017. Available: https://www.r-project.org/

45. Carpenter B, Gelman A, Hoffman MD, Lee D, Goodrich B, Betancourt M, et al. Stan: A Probabilistic Programming Language. J Stat Softw. 2017;76. doi:10.18637/jss.v076.i01

46. Stan Development Team. RStan: the R interface to Stan: http://mc-stan.org [Internet]. 2017.

Figure 1. Examples of accelerometer and GPS data recorded during solo and paired

flights. A, Bird S30 carrying an accelerometer (top) and GPS sensor (bottom) attached via Velcro strips. B-E, Dorsal body (DB) acceleration recorded by the accelerometer during S30's final release in each of the four conditions: $\mathbf{B}$, solo flight (blue); $\mathbf{C}$, paired flight with a similarsized bird (red); D, paired flight with a different-sized bird (dark red) and $\mathbf{E}$, solo flight (dark blue). Accelerometer data has been filtered and gravity removed (see Methods). Note the higher wingbeat frequency when the bird is flying in a pair. F, Routes flown by S30 during the final release of each of the four conditions (same flights as those shown in B-E). Note the straighter trajectory, and hence greater route accuracy, of the paired flights. Black circle corresponds to the release site and white circle corresponds to the home loft. Map designed 
761 using ArcGIS 10.4.1 (Esri Inc., Redlands, USA) using the World Topographic Map[23]. Scale

762 bar shows $3 \mathrm{~km}$.

763

764 Figure 2. Wingbeat frequency as a function of flight number. A-D, Median wingbeat

765 frequency (raw data with no covariates) for all 20 birds during each of the four experimental

766 phases: A, six individual releases (solo); B, six releases with a similar-sized bird (similar-sized

767 pair); $\mathbf{C}$, six releases with a different-sized bird (different-sized pair); and $\mathbf{D}$, six individual

768 releases (solo). E-H, Raw wingbeat frequency data for birds which flew in a pair $(<50 \mathrm{~m}$

769 distance) and solo (> $300 \mathrm{~m}$ ) during the same flight. $\mathbf{E}$ and $\mathbf{G}$ are from the similar sized pair of

770 birds B01 and B82, respectively (pictured). F and $\mathbf{H}$ are from the different-sized pair B01 and

771 B07 (pictured). Boxplots show the median and upper and lower quartiles, and whiskers

772 correspond to 1.5 times the interquartile range.

773

774 Figure 3. Boxplot of median vertical peak-to-peak head displacement across six birds, each

775 flying once solo and once in a pair. Bottom and top edges of the box indicate the 25th and 75th

776 percentiles and the whiskers extend to the most extreme data points. Note that there is no overlap between the median head displacements in the two conditions. 


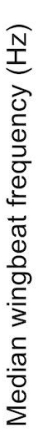

ำ

A

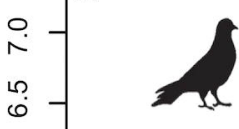

i

$\stackrel{0}{\circ}$
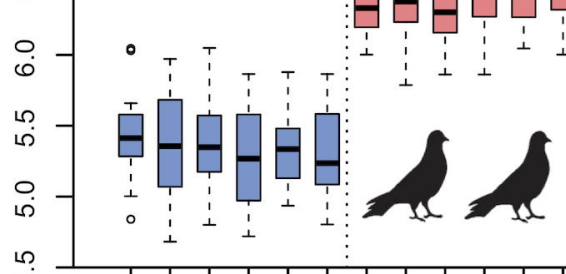

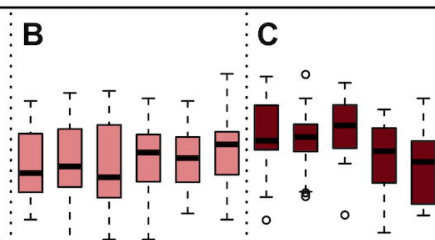

(

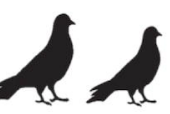

D
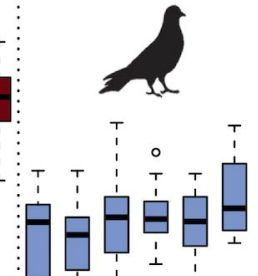

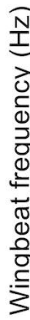

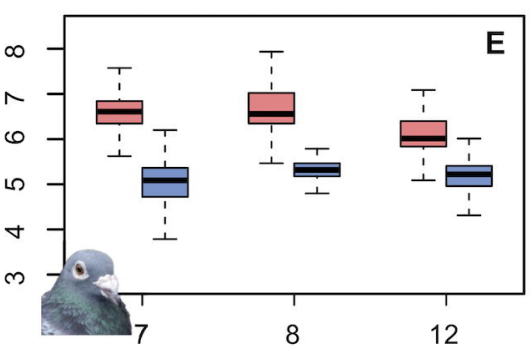

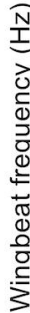

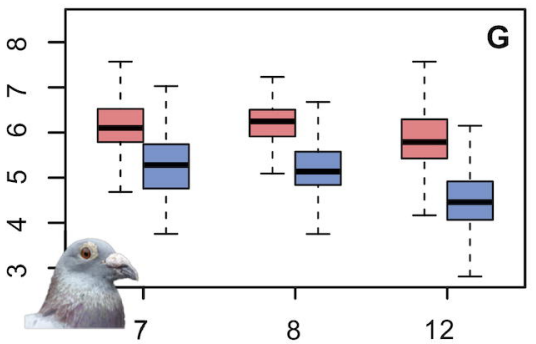

$\begin{array}{llllll}13 & 15 & 17 & 19 & 21 & 23\end{array}$
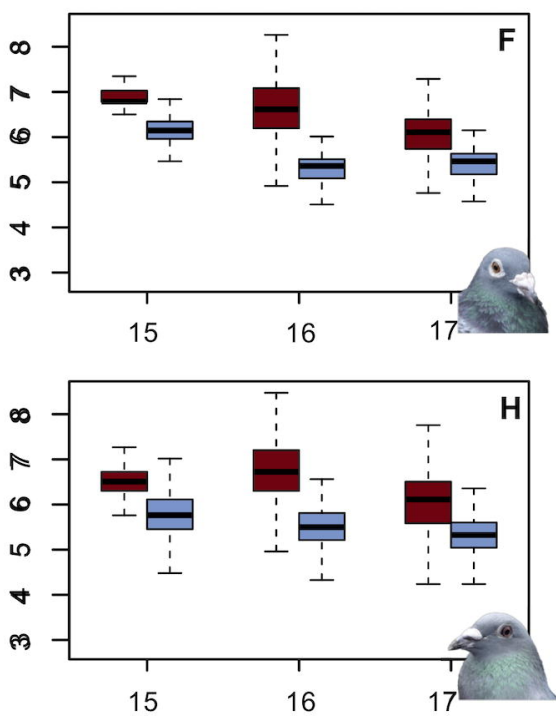

Flight number 
